\title{
A Model to Evaluate Smart Growth of a City Using AHP and CRITIC
}

\author{
Jing-Yan Li \\ School of North China Electric Power University BaoDing, Hebei 071000, China \\ 15932022562@163.com
}

Keywords: smart growth, Analytic Hierarchy Process, CRITIC

\begin{abstract}
With the development of the society, smart growth of a city is becoming more and more popular. Whether a city grows smart is becoming an important criterion to evaluate its advancement. This paper firstly formulates Smart Growth Indicator (SGI) using Analytic Hierarchy Process (AHP) combined with CRITIC to account for the wide variety of factors that influence smart growth of a city. Then, to evaluate the sensitivity of the model, data of Arlington are collected. Results shows that the model works very well.
\end{abstract}

\section{Introduction}

Smart growth is a concept in contrast to sprawl development which embraces economically prosperous, socially equitable, and environmentally sustainable. The concept derives from the United States initially, and implementing very well in a lot of cities in America, such as Denver and Arlington [1].

In order to devise a valuable model to evaluate smart growth, firstly, considering the repeatability of information and the unnecessary workload of our work, this paper choose Principle Component Analysis (PCA) to make dimensionality reduction. Next, CRITIC and Analytic Hierarchy Process (AHP) were invoked to calculate the comprehensive weight of the selected indicators for the sake of getting the most reasonable weight. Finally, in illustrating the success of the plan, this paper propose two criterions respectively allowing for the time and space which are respectively called average growth rate (AGR) and average pulling rate (APR). Only both the AGR and APR values are greater than zero can we believe that the current plan is successful.

\section{Selection of Indicators}

\section{Initial Selection of Indicators.}

Based on widely reading and investigating the 10 principles of smart growth, a set of 21 indictors of smart growth were defined for three categories: environment, social, and economic. The indictors were generally thought to influence or correlate with the condition of smart growth of a city.

The specific indicators were shown as Fig.1.

\section{Data preprocessing.}

According to the $n$ indicators in a dimension, we select $m$ years' number of samples to construct data matrix $\mathrm{X}=\left[X_{i j}\right](\mathrm{i}=1,2, \ldots, \mathrm{m} ; \mathrm{j}=1,2, \ldots, \mathrm{n})$. Next, we use dimensionless method to process the original data.

$$
\begin{array}{ll}
\mathrm{b}_{\mathrm{ij}}=\left(x_{i j}-\overline{x_{j}}\right) / S_{j} & \text { (positive indicator) } \\
\mathrm{b}_{\mathrm{ij}}=\left(\overline{x_{j}}-x_{i j}\right) / S_{j} & \text { (negative indicator) }
\end{array}
$$

Where $\overline{x_{j}}=\frac{1}{m} \sum_{i=1}^{m} x_{i j}, S_{j}=\frac{1}{m-1} \sqrt{\sum_{i=1}^{m}\left(x_{i j}-\overline{x_{j}}\right)^{2}}$. Then, we can get a new dimensionless matrix $\boldsymbol{B}=\left[\mathrm{b}_{\mathrm{ij}}\right]$ 


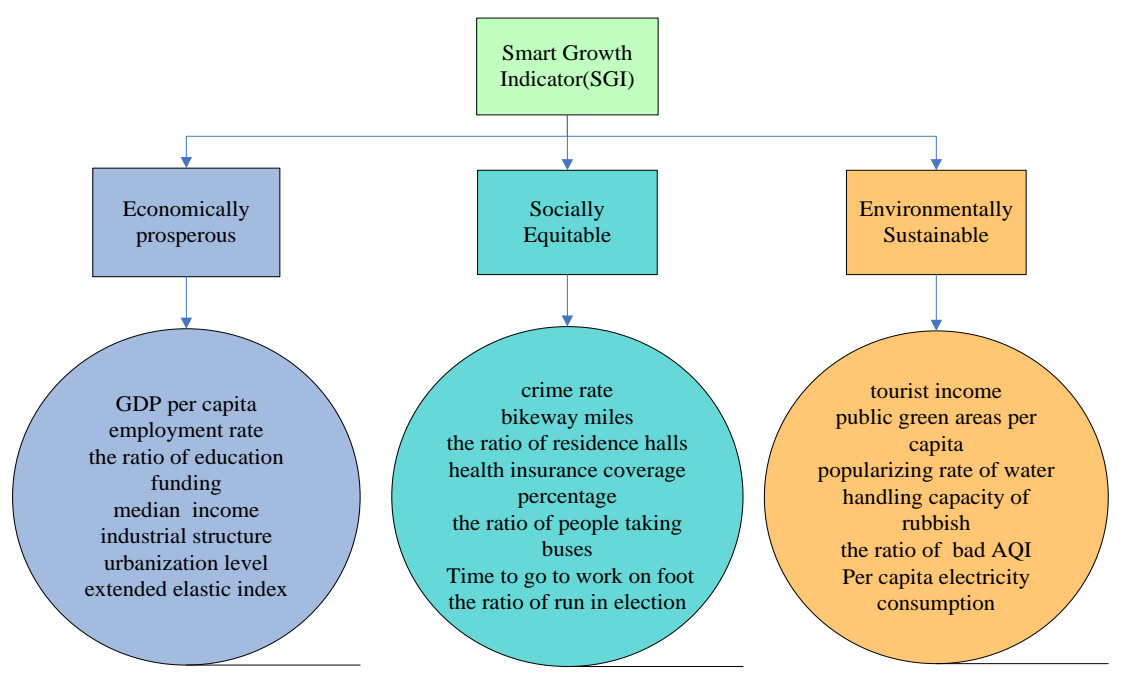

Fig. 1 the specific indicators we selected

\section{Determination of Indicators.}

Then, the correlation coefficient should be calculated.

$$
r_{i j}=\frac{1}{m-1} \sum_{k=1}^{m} b_{k i} b_{k j},(i, j=1,2, \ldots, n)
$$

Where $r_{i j}$ is the correlation coefficient of $i$ and $j$, the parameters $b_{k i}$ and $b_{k j}$ are standardized data.

Then, we calculate the characteristic value $\lambda_{\mathrm{k}}(\mathrm{k}=1,2, \ldots \mathrm{n})$ of matrix $\mathbf{R}$ and characteristic vector $\left(u_{1}, u_{2}, \ldots, u_{\mathrm{n}}\right)$, where $\mathrm{u}_{\mathrm{ij}}=\left(\mathrm{u}_{1 \mathrm{j}}, \mathrm{u}_{2 \mathrm{j}}, \ldots, \mathrm{u}_{\mathrm{mj}}\right)^{\mathrm{T}}$ is the $m$ th principal component consisting of characteristic vector.

Next, ranking the characteristic value and calculating the rate of contribution $\boldsymbol{T}_{\mathrm{k}}$ and then accumulating contribution rate $\boldsymbol{D}_{\mathrm{k}}$ of k indicators.

$$
\begin{gathered}
T_{k}=\lambda_{k} / \sum_{k=1}^{n} \lambda_{k} \\
D_{k}=\sum_{k=1}^{n} T_{k}
\end{gathered}
$$

Selecting the $p$ principal components which satisfy that $\boldsymbol{D}_{\mathrm{p}} \geqslant 85 \%$ :

$$
\begin{aligned}
& y_{1}=u_{11} b_{1}+u_{21} b_{2}+\cdots+u_{n 1} b_{n} \\
& y_{2}=u_{12} b_{1}+u_{22} b_{2}+\cdots+u_{n 2} b_{n} \\
& \cdots \\
& y_{p}=u_{1 p} b_{1}+u_{2 p} b_{2}+\cdots+u_{n p} b_{n}
\end{aligned}
$$

Additional, we proceed to observe the characteristic vector of principal components and select the indicator which has a bigger characteristic value and then we finish the selection of the indicators.

\section{Weight Modification with AHP and CRITIC.}

Considering that each city has its own development situation, thus we cannot assign the same weight on different cities. In order to get the most rational weight, we adopt Analytic Hierarchy Process (AHP) and CRITIC to calculate the weight of the indicators. Details are as follows:

\section{$\checkmark$ STEP 1: Subjective Weight Analysis By AHP}

Analytic Hierarchy Process (AHP) is a kind of decision analysis method which combines qualitative and quantitative analysis to solve complex problems with multiple objectives. It aims at judging the importance degree between the two indicators [2]. Then, by checking the consistency of the pairwise matrices, we can minimize the bias from subjective factors, thus providing a more reasonable and convincing evaluation scheme [3]. The specific hierarchical graph can be seen as above Fig.1.

And then we can get the weight vector $W_{\mathrm{i}}{ }^{\prime}(\mathrm{i}=1,2, \ldots, \mathrm{k})$ of the indicators layer to destination layer. 


\section{$\checkmark$ STEP 2: Objective Weight Analysis By CRITIC Method}

CRITIC (Criteria Importance through Inter-Criteria Correlation) is an objective weight determination method proposed by Diakoulaki [4]. The specific steps are as follows:

Firstly, calculating the conflict quantitative index of the indicator $j$ to other indicators.

$$
C_{\mathrm{j}}=\sum_{i=1}^{k}\left(1-r_{i j}\right)
$$

Where $\mathrm{r}_{\mathrm{ij}}$ represents the correlation index between $i$ and $j$. The bigger $\mathrm{C}_{\mathrm{j}}$ is, the more information the indicator contains, and more important the indicator is.

The information indicator $j$ contains are as follows:

$$
G_{j}=S_{j} C_{j}
$$

Where, $\mathrm{S}_{\mathrm{j}}$ represents the standard deviation of indicator $j$. The calculated mode of $\mathrm{S}_{\mathrm{j}}$ can be expressed as:

$$
S_{j}=\frac{1}{k-1} \sqrt{\sum_{i=1}^{k}\left(b_{i j}-\overline{x_{j}}\right)^{2}}
$$

Then, the mean value of indicator $j$ is:

$$
\overline{X_{j}}=\sum_{i=1}^{k} b_{i j} / k
$$

The normalization coefficient of indicator $j$ is:

$$
W_{j}=G_{j} / \sum_{j=1}^{k} G_{j}
$$

Then, we can obtain the coefficient calculated by CRITIC. To put into simplify, we define $\mathrm{W}_{\mathrm{j}}=\mathrm{W}_{\mathrm{i}}{ }^{\prime}{ }^{\prime}(\mathrm{i}=1,2, \ldots, \mathrm{k})$

Finally, we can get the comprehensive weight:

$$
\mathrm{w}_{\mathrm{i}}=\alpha \mathrm{w}_{\mathrm{i}}{ }^{\prime}+(1-\alpha) \mathrm{w}_{\mathrm{i}}{ }^{\prime \prime},(\mathrm{i}=1,2, \ldots \mathrm{k})
$$

Where $\alpha$ represents the different assignments of the weights in the coefficient of AHP and CRITIC. In this paper, we decide to make $\alpha=0.5$. Afterwards, we will make an sensitivity analysis of it.

\section{Determination of the Final Metric.}

By above calculating, we can get the comprehensive evaluation metric, i.e. SGI:

$$
S G I=\sum_{i=1}^{k} w_{i} b_{i}
$$

Where, $\mathrm{b}_{\mathrm{i}}$ represents the $i$ th principle component.

Next, allowing for that different city have different situation, therefore we cannot evaluation the success of a city by only an absolute number. In our opinion, the success of a plan must consider two dimensions: time and space. To be specific, the time refers to that the city must develop better and better, in other words, the value of SGI in recent period must bigger than the previous period. And the space refers to that the pulling function of the city to the local province (China) or state (America) must be better and better. Based on the above consideration and in order to acquire more accurate results, we transform SGI to AGR (Average Growth Rate) and APR (Average Pulling Rate).

$$
\begin{gathered}
A G R=\frac{1}{p} \sum_{i=p+1}^{2 p} \frac{S G I_{i+1}-S G I_{i}}{S G I_{i}}-\frac{1}{p} \sum_{i=1}^{p} \frac{S G I_{i+1}-S G I_{i}}{S G I_{i}} \\
A P R=\frac{1}{p} \sum_{i=p+1}^{2 p} \frac{S G I_{i}-S G I_{i}{ }^{\prime}}{S G I_{i}{ }^{\prime}}-\frac{1}{p} \sum_{i=1}^{p} \frac{S G I_{i}-S G I_{i}{ }^{\prime}}{S G I_{i}{ }^{\prime}}
\end{gathered}
$$

Where $p$ refers to the period we study includes $p$ years. 
Finally, a metric to measure smart growth of a city is defined:

$$
\text { metric }=\left\{\begin{array}{l}
\text { success, } A G R>0, A P R>0 \\
\text { unsuccess, others }
\end{array}\right.
$$

\section{Sensitivity Analysis}

In the process of determining the comprehensive weight, we introduce $\alpha$ to control it. To validate the effectiveness of our model, we search some relevant data of 2015 about Arlington to test the sensitivity of $\alpha$. We very $\alpha$ from $0.3-0.7$ and then we observe the trend of the value SQI. The results are as Fig. 2.

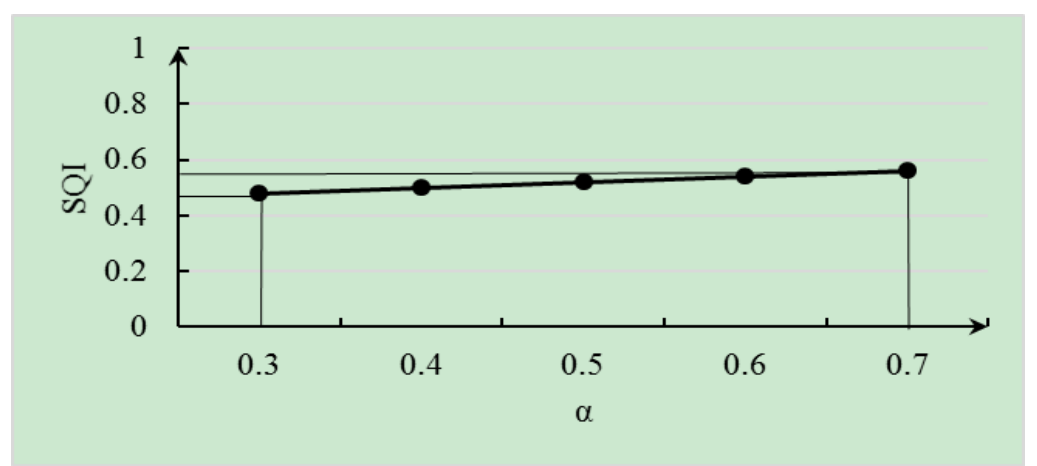

Fig 2 the value of SQI

As shown in Fig. 2, we find that the value of SGI vary slowly, which means that it is very stable. Thus, we can get the conclusion that the model performs very well.

\section{Conclusions}

In this paper, firstly, we started with devising a new indicator SGI using AHP and CRITIC accounting for the wide variety of factors that influence smart growth of a city, then introducing two criterions to judge the success of it, respectively called AGR and APR from the perspective of time and space. Ultimately, we make sensitivity analysis on $\alpha$ to validate the effectiveness of our metric. Results show that it performs very well.

\section{References}

[1] Wikipedia, 'Smart Growth', Information on https://en.wikipedia.org/wiki/Smart_growth

[2] DING Xiao-Qin, and ZHANG De-Sheng. "K-Means Algorithm Based on Synthetic Weighting of AHP and CRITIC," Computer Systems \& Applications, vol. 7, pp 182-186, 2016.

[3] Kurttila, Mikko, et al. "Utilizing the analytic hierarchy process (AHP) in SWOT analysis - a hybrid method and its application to a forest-certification case." Forest Policy \& Economics, vol. 1, No.1, pp 41-52, 2000.

[4] WANG Yuan, WU Ze-min, "Landscape pattern analysis and comprehensive assessment of urban forest in the three districts of Maanshan City based on RS and GIS," Journal of Beijing Forestry, vol.30, No.4, pp 46-52, 2008. 